\title{
Influence of a Character-Based App on Children's Learning of Nutritional Information: Should Apps Be Served with a Side of Media Characters?
}

\author{
Marisa M. Putnam, MPP, Elana M. Richmond, BA, Kaitlin L. Brunick, PhD, \\ Charlotte A. Wright, BA, and Sandra L. Calvert, PhD
}

\begin{abstract}
Objective: Childhood obesity is a health issue in the United States, associated with marketing practices in which media characters are often used to sell unhealthy products. This study examined exposure to a socially contingent touch-screen gaming app, which replied immediately, reliably, and accurately to children's actions. Children's recall of nutritional content and their liking of the character were assessed.

Materials and Methods: Four- and five-year-old children $(N=114)$ received no-exposure, single-exposure, or repeated-exposure to a character-based iPad app rewarding healthy and penalizing unhealthy behaviors. Children reported how much they liked the character and recalled healthy and unhealthy items from the app. An ordinary least squares regression was conducted on how much children liked the character by condition. Poisson regressions were conducted on the number of items recalled by condition alone, and in an interacted model of treatment condition by liking the character.

Results: Children liked the character more in the repeated app-exposure condition than in the control group $(P=0.018)$. Children in the repeated and single app-exposure conditions recalled more healthy $(P<0.001)$ and unhealthy $(P<0.001)$ items than the control group. Within treatment conditions, liking the character increased recall of healthy items in the single app-exposure compared to the repeated app-exposure condition $(P=0.005)$. Conclusions: Results revealed that repeated exposure increased children's learning of nutritional information and liking of the character. The results contribute to our understanding of how to deliver effective nutrition information to young children in a new venue, a gaming app.
\end{abstract}

Keywords: Food marketing, Obesity, Young children, Media characters, Apps, Nutrition

\section{Introduction}

C HILDHOOD OBESITY IS a serious health issue in the United States, affecting $\sim 17 \%$ of children, which has persisted despite clinical and public health interventions. ${ }^{1}$ An Institute of Medicine review found an association between childhood obesity and child-targeted marketing practices, which include media characters that market unhealthy foods and beverages to children. ${ }^{2}$ Media characters in the 21 st century do not simply sell unhealthy products like sugary cereals ${ }^{3}$ on traditional media platforms like television. The lines between advertisement and content have become increasingly blurred as products are placed within digital games alongside popular media characters. ${ }^{4}$ With the rise of daily mobile device usage for children, ${ }^{5}$ it is important to understand how learning occurs in this digital setting. ${ }^{5}$ Our purpose was to examine (1) whether liking a character develops through app-play, and (2) how a liked character within a socially contingent touch-screen gaming app influences recall of nutritional information.

Children's liking of characters influences cognitions and behaviors, ${ }^{6}$ and is considered a major component of viewer reactions toward characters. ${ }^{7}$ For adults, liking a media character is associated with perceiving the character as a role $\operatorname{model}^{8}$ and increased efforts to imitate the character. ${ }^{9}$ Similarly, adults are more involved with characters that they like versus dislike, which includes motivations to imitate behaviors that characters are rewarded for. ${ }^{10}$ Children are more likely to select the same snack, healthy or unhealthy,

(C) Marisa M. Putnam et al., 2018; Published by Mary Ann Liebert, Inc. This Open Access article is distributed under the terms of the Creative Commons Attribution Noncommercial License (http://creativecommons.org/licenses/by-nc/4.0/) which permits any noncommercial use, distribution, and reproduction in any medium, provided the original author(s) and the source are credited. 
that a popular character holds ${ }^{11}$ or consumes. ${ }^{12}$ Liking characters may be one reason why children are more likely to focus their attention on, ${ }^{13}$ and select products, ${ }^{14}$ associated with popular media characters; this potentially could lead to increased learning when the characters are contingently rewarded or punished.

Serious games for health can influence players' healthrelated behaviors when they experience interactivity, or cause-and-effect relationships based on their actions. ${ }^{15}$ Apps on touch-screen devices consistently and accurately respond to an individual's touch of a specific location on the screen, allowing interactivity on command. A game-based app featuring a character responds in a socially contingent manner, since a media character embedded within the app responds to a child's actions immediately, reliably, and accurately. ${ }^{16}$ The response may be in the form of rewards and punishments. For example, 9- and 10-year-olds were more likely to select and consume foods and beverages, healthy or unhealthy, if their Pac-Man character had been contingently rewarded or punished for snack selections when playing a Pac-Man videogame. ${ }^{12}$ Digital games and apps allow children repeated access to these socially contingent interactions with media characters, delivering age and content appropriate information from essentially anywhere.

Repeated exposure leads to the mere exposure effect, in which individuals increase their liking and preference for things they are familiar with, ${ }^{4,17,18}$ in our case, a media character in an app. Repeated exposure to messages also improves learning, which is particularly important for children under age eight who have limited cognitive resources to process messages, ${ }^{4}$ including those that market products to them. For example, children were more likely to remember a product when they viewed a commercial repeatedly, ${ }^{4}$ and young adults recalled more information from a television program when they were frequent viewers of the series. ${ }^{19}$

The current study examined (1) how liking a media character in an app develops, and (2) if young children's recall of nutritional information is influenced by the amount of exposure to the app, particularly if they like the character. Two treatment conditions were exposed to an iPad app featuring a character for either 5 days or a single 31-minute play session. A control group was not exposed to the app. We measured how much children liked the character and their free recall of nutritional information in the app. We hypothesized the following:

(1) children in the repeated app-exposure condition will like the character more than children in the single app-exposure or no exposure conditions;

(2) children in the repeated app-exposure condition will recall more healthy and unhealthy foods and beverages from the app than the single app-exposure and no app-exposure control groups, and those in the single app-exposure condition will recall more items than the control group;

(3) within the treatment conditions, the more children like the character, the more healthy and unhealthy foods and beverages from the app they will recall.

\section{Materials and Methods}

\section{Participants}

One-hundred fourteen 4 - and 5-year-old children $\left(M_{\text {years }}=\right.$ $4.99, S D=0.64 ; 50 \%$ girls) were recruited via email from a lab-managed database of children in a large metropolitan area. The university IRB approved the study protocol. Parents provided written consent and demographic information, while children verbally assented to participate in the study.

\section{D.W.'s ${ }^{\mathrm{TM}}$ Unicorn Adventure App}

The goal of the app was to help D.W., the preschool-aged younger sister of the main character in the educational PBS cartoon Arthur, navigate 12 levels of increasing difficulty to help a lost unicorn return home (Fig. 1). To fuel their appadventure, children helped D.W. "eat" 12 energy-packed fruit and vegetable snacks and avoid six energy-zapping junk snacks. The app contingently rewarded children with points when children helped D.W. eat healthy items and subtracted points when children had D.W. consume unhealthy ones. Single-and double-tapping gestures were required to navigate the game, which was played on an iPad 2 with a $9.5^{\prime \prime}$ by $7.31^{\prime \prime}$ screen.

\section{Experimental procedure}

Within sex, children were randomly assigned to one of three conditions: repeated app-exposure, single app-exposure, or a no app-exposure control group. Trained experimenters conducted the study at participants' homes. Children in the repeated app-exposure condition received an iPad with access to only D.W.'s Unicorn Adventure to play for 5 days as much as they desired. Parents were contacted daily and asked whether their child played the app that day.

On day 5, children in the repeated app-exposure condition played the app for a maximum of 31 minutes and then answered measures presented by experimenters. Children in the single app-exposure condition were visited once by experimenters, played the app for up to 31 minutes, and then answered measures. Children in the no app-exposure control group were visited once by experimenters, and only answered measures. All final sessions were videotaped to measure and report gameplay time.

\section{Measures}

To assess children's liking of the character, children were first shown an 8.5 " by 11 " picture of D.W. and asked, "How much do you like D.W. ${ }^{\text {TM}}$ ?" The experimenter pointed at a 5point smiley face Likert scale and labeled the faces as "not at all," "a little," "kind of," "a lot," and "a whole lot." Children selected an answer by pointing to a smiley face, saying their reply, or a combination of the two responses. This procedure was adapted from previous studies with adults. ${ }^{8-10}$

Children's free recall of healthy and unhealthy items from the app was assessed using procedures adapted from previous studies ${ }^{20}$ in which children recalled information from a mov$\mathrm{ie}^{18}$ or a computer screen. ${ }^{21,22}$ In the current study, children were asked: "What foods are healthy for D.W. ${ }^{\mathrm{TM}}$ and give her energy?" "What foods are unhealthy for D.W. ${ }^{\mathrm{TM}}$ and take her energy away?" Children's responses were transcribed and coded to assess whether the healthy and unhealthy items recalled were present in the app. Children received one point for each healthy item recalled; these points were summed to make a total healthy item recall score $(M=2.18, S D=2.03$, range 0-9). The same method was used to calculate the number of unhealthy items recalled $(M=1.18, S D=1.36$, 


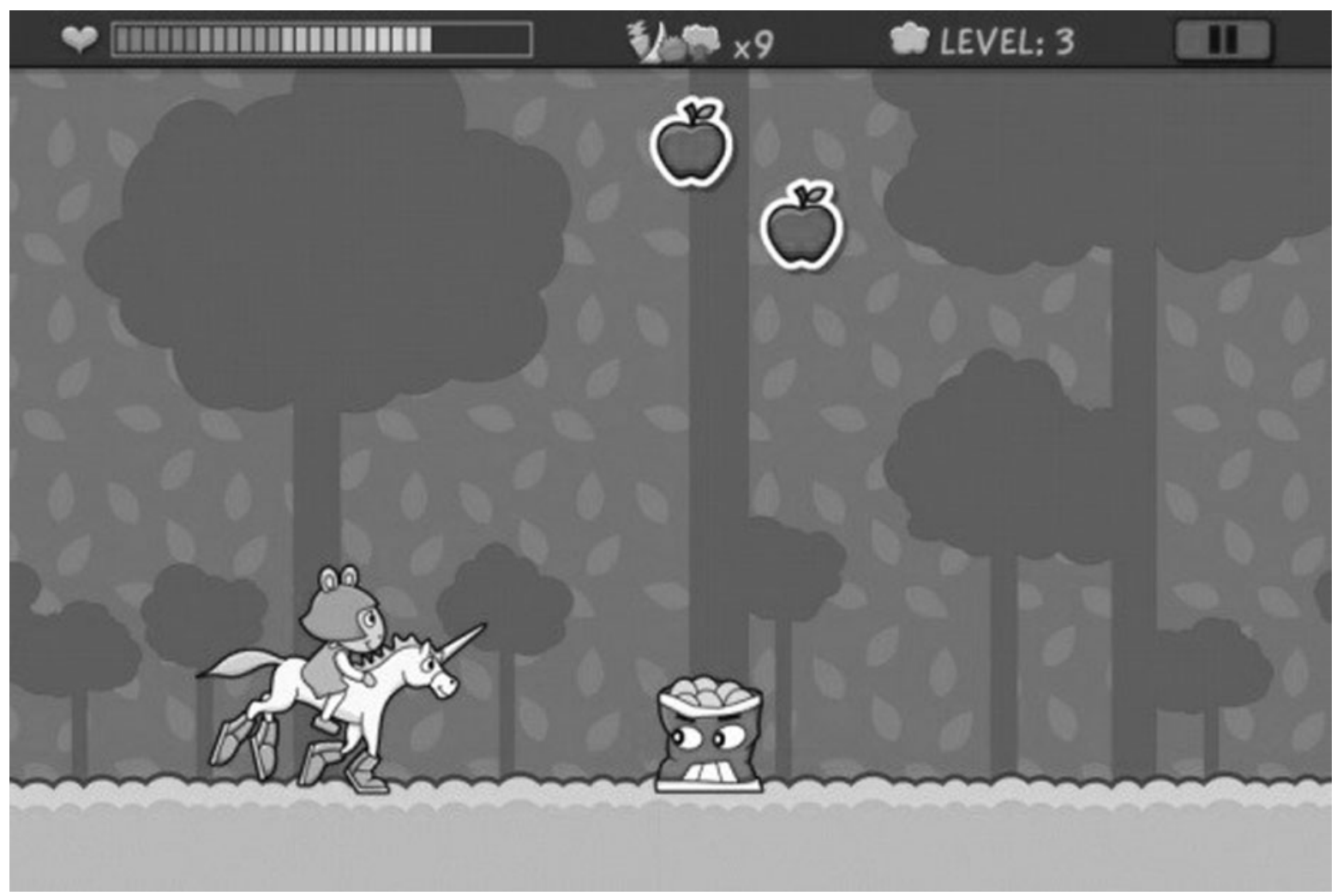

FIG. 1. Screenshot of one world in D.W.'s Unicorn Adventure app.

range 0-6). For reliability, two trained research assistants calculated the number of healthy and unhealthy items recalled for $20 \%$ of the sample (Cohen's Kappa for healthy items $=1.0$; Cohen's Kappa for unhealthy items $=0.78$ ).

\section{Data analysis plan}

To examine hypothesis 1 , an ordinary least squares (OLS) regression was run to determine whether children in the repeated app-exposure condition reported liking the character more than the control or single app-exposure conditions. Because the number of healthy and unhealthy items recalled involved count variables, Poisson regression models, interpreted with incidence rate ratios, were selected to examine hypotheses 2 and $3 .^{23}$ For hypothesis 2 , Poisson regressions were run to examine condition differences in the number of healthy and unhealthy items recalled, respectively. For hypothesis 3 , Poisson regression analyses were run to examine differences in the number of healthy and unhealthy items recalled, respectively, for the single and repeated appexposure treatment conditions as a function of liking the character, centered at the mean value of 3.07. One model examined the individual contributions of treatment condition and liking the character. A second model included the interaction of treatment condition and liking the character. All results were evaluated with Bonferroni adjusted alpha levels.

\section{Results}

The sample $(N=114)$ was composed of Caucasian $(64.0 \%)$, other or mixed-race $(24.6 \%)$, African American
(7.9\%), Asian (2.6\%), and Hispanic (0.9\%) children. Parents' reported education levels of a high school diploma $(0.9 \%)$, some college $(3.5 \%)$, college degree $(24.6 \%)$, and a postgraduate school/professional degree $(71.1 \%)$. Ten additional children were excluded from the sample due to experimenter error $(n=2)$, refusal to play the app in treatment conditions $(n=6)$, or refusal to answer survey questions $(n=2)$. The excluded children did not differ significantly from the remaining sample by age, gender, ethnicity, parental education, or condition.

According to parents, all children in the repeated appexposure condition played the app over the course of 5 days (range of 2-5 days). The frequency of play was $100 \%$ on day 1 , $75 \%$ on day $2,83 \%$ on day $3,78 \%$ on day 4 , and $74 \%$ on day 5 .

Children in the treatment conditions played the app on the test day for $\sim 11$ minutes $(M=11.46, S D=10.25$ minutes; range $=0.52-31.38$ minutes $)$. The amount of time playing the app did not differ significantly by treatment condition.

An OLS regression revealed that liking D.W. differed significantly by condition, $F(2,111)=3.07, P=0.05, R^{2}=0.05$. As predicted, children in the repeated app-exposure condition $(M=3.58, S D=1.44)$ liked D.W. significantly more than the control group did $(M=2.73, S D=1.58), t(111)=-2.47$, $P=0.018$, which met a Bonferroni adjusted alpha value of 0.025 for this model. Contrary to prediction, the repeated appexposure condition did not significantly differ from the single app-exposure condition $(M=2.95, S D=1.62)$.

Table 1, which displays incidence rate ratio (IRR) results of a Poisson regression analysis for free recall of healthy items in the app, supports hypothesis 2 . For children in the single appexposure condition group $(M=2.08, S D=1.55)$, the incidence 
Table 1. Poisson Regressions Predicting Free Recall of Healthy and Unhealthy Items BY CONDITION $(N=114)$

\begin{tabular}{lccc}
\hline & Healthy items & & Unhealthy items \\
Variable & IRR & & IRR \\
\hline Condition & & \\
Control group & - & - \\
Single app-exposure & $1.85^{*}(0.35)$ & $3.79^{*}(1.24)$ \\
Repeated app-exposure & $3.09^{*}(0.53)$ & $7.69^{*}(2.38)$ \\
LR $X^{2}$ & $49.14^{*}$ & $66.98^{*}$ \\
McFadden's Pseudo $R^{2 \mathrm{~b}}$ & 0.10 & 0.19 \\
\hline
\end{tabular}

Standard errors are in parentheses.

$* P \leq 0.017$.

${ }^{\text {a } E a c h ~ c o n d i t i o n ~ i s ~ d u m m y ~ c o d e d ~ a s ~} 1$, the control group is the omitted reference category and coded as 0 .

${ }^{b}$ McFadden s Pseudo $R^{2}$ is a measure of the model's goodness of fit. IRR, incidence rate ratio.

rate ratio of the number of healthy items recalled increased significantly by a factor of 1.85 compared to the control group $(M=1.12, S D=1.42), P=0.001$. The incidence rate ratio of free recall of an additional healthy food or beverage is significant at 3.09 times higher for children in the repeated-app exposure condition $(M=3.47, S D=2.35)$ compared to the control group, $P<0.001$. We also tested whether the incidence rate ratios of the single and repeated app-exposure conditions were equal to each other. For children in the single appexposure condition, the incidence rate ratio of the number of healthy items recalled decreased significantly by a factor of 0.51 compared to the repeated app-exposure condition, $P<0.001$. The alpha values in these comparisons met Bonferroni corrected alpha values of 0.017 .

Table 1, which displays the incidence rate ratios of a Poisson regression analysis for free recall of unhealthy items in the app, also supports hypothesis 2 . For children in the single app-exposure condition $(M=1.11, S D=1.07)$, the incidence rate ratio of the number of unhealthy items recalled increased significantly by a factor of 3.79 compared to the control group $(M=0.29, S D=0.46), P<0.001$. The incidence rate ratio of free recall of an additional unhealthy food or beverage was significant at 7.69 times higher for children in the repeated-app exposure condition $(M=2.25, S D=1.57)$ compared to the control group, $P<0.001$. We also tested whether the incidence rate ratios of the single and repeated app-exposure conditions were equal to each other. For children in the single app-exposure condition, the incidence rate ratio of the number of unhealthy items recalled decreased significantly by a factor of 0.71 compared to the repeated app-exposure condition, $P<0.001$. The alpha values in these comparisons met Bonferroni corrected alpha values of 0.017.

Table 2 displays the incidence rate ratios of a hierarchical Poisson regression analysis for recall of healthy items in the app predicted by treatment condition and liking of D.W., and partially supported hypothesis 3 . In model 1 , results revealed that for children in the single app-exposure group, the incidence rate ratio for number of healthy items recalled decreased by a factor of 0.60 compared to the repeated exposure condition, controlling for liking D.W., $P=0.001$. In model 2 , the interaction of treatment condition and liking D.W. revealed that as liking D.W. increased above the mean in the single app-exposure condition, the incidence rate ratio for recall of healthy items increased by a factor of 1.30 compared to the repeated appexposure condition, $P=0.005$. The incidence rate ratio of recalling healthy items in the single app-exposure condition decreased by a factor of 0.057 compared to the repeated appexposure condition, at children's average reported liking of D.W., $P<0.001$. In other words, children recalled healthy items at a higher rate in the repeated app-exposure compared to the single app-exposure condition for children who liked the character an average amount. The results were robust to Bonferroni corrected alpha values of 0.017 .

Table 2 also displays the incidence rate ratios of a hierarchical Poisson regression analysis for recall of unhealthy items in the app predicted by treatment condition and liking D.W. In model 1, results revealed that for children in the single app-exposure condition, the incidence rate ratio for number of unhealthy items recalled decreased significantly by a factor of 0.49 compared to the repeated app-exposure condition, holding liking D.W. constant, $P<0.001$, which was robust to a Bonferroni adjusted alpha value of 0.025 . Contrary to hypothesis 3 , the interaction of treatment condition and liking D.W. revealed no significant interactions or simple effects on the number of unhealthy items recalled.

\section{Discussion}

Children encounter media characters marketing foods and beverages through practices that increasingly blur the

Table 2. Poisson Regressions Predicting Free Recall of Healthy and Unhealthy Items By Treatment Condition and Liking D.W. $(N=73)$

\begin{tabular}{|c|c|c|c|c|}
\hline \multirow{3}{*}{$\frac{\text { Variable }}{\text { Single app-exposure }^{a}}$} & \multicolumn{2}{|c|}{ Healthy items } & \multicolumn{2}{|c|}{ Unhealthy items } \\
\hline & \multicolumn{2}{|c|}{$\operatorname{IRR}$} & \multicolumn{2}{|c|}{$\operatorname{IRR}$} \\
\hline & $0.60 *(0.09)$ & $0.57 *(0.08)$ & $0.49 *(0.10)$ & $0.46(0.20)$ \\
\hline Liking D.W. b $^{\mathrm{t}}$ & $1.01(0.05)$ & $0.90(0.06)$ & $0.99(0.06)$ & $0.98(0.08)$ \\
\hline Single app-exposure $\times$ Liking D.W. & - & $1.30 *(0.12)$ & - & $1.02(0.12)$ \\
\hline $\operatorname{LR} X^{2}$ & $12.91 *$ & $20.67 *$ & $14.51 *$ & $14.54 *$ \\
\hline McFadden's Pseudo $R^{2 \mathrm{c}}$ & 0.04 & 0.07 & 0.06 & 0.06 \\
\hline
\end{tabular}

Standard errors are in parentheses.

$* P \leq 0.017$.

${ }^{\mathrm{a}}$ The omitted reference category is the repeated app-exposure condition.

${ }^{\mathrm{b}}$ Liking D.W. is mean-centered at 3.07.

'McFadden's Pseudo $R^{2}$ is a measure of the model's goodness of fit. 
lines between fun game content and marketed messages. ${ }^{4}$ Character-based marketing practices often sponsor products that are low in nutritional content ${ }^{2}$ and can influence children's preferences and consumption of foods and beverages. ${ }^{2}$ Little is known about young children's recall of nutritional information from a character-focused app, which contingently rewards healthy choices and penalizes unhealthy choices.

This study provides evidence to support the mere exposure effect ${ }^{17,18}$ for liking a character. Specifically, children reported liking the character more after exposure to an app over 5 days when compared to the no exposure control group. These data provide evidence that app play can increase children's liking of characters who deliver messages to them.

This study also contributes to the growing literature on the relationship between socially contingent interactions and learning in digital game settings. The social contingency afforded by a character in the touch-screen app, who was rewarded for healthy behavior and penalized for unhealthy behavior, led to increased recall of both healthy and unhealthy items with more exposure to the app. This finding is consistent with a socially contingent game influencing snack selection and consumption for older 9- and 10-year-old children. ${ }^{12}$ Mobile apps and games providing contingent interactions are easily played repeatedly and on demand, and may complement more traditional educational opportunities for nutritional education. ${ }^{24}$

The combination of liking the character and social contingency significantly improved recall of nutritional knowledge when children had only a single session of exposure to the app. For each point higher children liked D.W. in the single app-exposure group, their recall for healthy items increased by over 1 item. Even when exposure is limited to half an hour, then, liking a character can result in better recall of targeted nutritional content. This finding mirrors the adult literature, in which liking a character increased efforts to imitate a character and perceptions of a character as a role model. ${ }^{8-10}$ This pattern was not supported in the repeated app-exposure condition, where liking the character more did not help children recall more items from the app. One explanation is that recall in the repeated app-exposure condition simply improved through repetition. Liking a character, though, may be a motivational gateway into playing an app in the future, thus providing repeated exposure to the nutritional information.

This study contributes to a body of research indicating that healthy games are effective tools from which children transfer information into their lives, ${ }^{12,25,26}$ in this case through liking and socially contingent interactions with a character. Apps for health may be an effective, relatively low-cost venue for children's nutrition education when a likeable character is featured.

A limitation of this study is that children's memory was measured soon after their last exposure to the app. Future research could explore children's recall of nutritional information after a delay. Another limitation is that the parents in this sample were highly educated, which could make these results less generalizable to other populations. Future research could also assess whether children's nutritional knowledge would lead them to modify their behavior when selecting and consuming actual snacks, particularly for overweight children who are more likely to be attentionally biased toward food images. $^{27}$
In conclusion, young children recall nutritional information when it is presented by a likeable media character in an app that uses socially contingent rewards and punishments. Therefore, we recommend that apps be served with a side of media characters using social contingency to teach children nutritional lessons during the app play that pervades many children's daily experiences.

\section{Acknowledgments}

The authors wish to thank Bill Shribman for allowing us to use the app in this study, Kevin Lesniewicz for technical assistance with the app, and Rebecca Ryan, $\mathrm{PhD}$, for assistance with the statistical analyses. The authors thank Naomi Aguiar, PhD, Elizabeth Mah, Brigid McCaffery, Genevieve Lai, Angella Liu, Elizabeth Fantini, Sana Charania, Marie Frolich, Molly Biedermann, Audra Fitzgerald, Lena Deb, Kate Shambaugh, Annabelle Heisley, and Nicole Schwab for assistance with data collection, coding, and feedback. The authors would especially like to thank the children, families, and childcare centers that participated in this study. This research was supported by a grant from the National Science Foundation (NSF-DLS Grant No. 1251745) to S.L.C. In addition, this material is based upon work supported by the National Science Foundation Graduate Research Fellowship Program under Grant No. DGE-1444316 to M.M.P. Any opinions, findings, and conclusions or recommendations expressed in this material are those of the author(s) and do not necessarily reflect the views of the National Science Foundation.

\section{Author Disclosure Statement}

No competing financial interests exist.

\section{References}

1. Skinner AC, Perrin EM, Skelton JA. Prevalence of obesity and severe obesity in US children, 1999-2014. Obesity 2016; 24:1116-1123.

2. McGinnis J, Gootman J, Kraak V. (Eds.) Food Marketing to Children and Youth: Threat or Opportunity IOM Report. Washington, DC: National Academies Press; 2006.

3. Galloway DP, Calvert SL. Media characters as spokespeople in U.S. grocery stores: Promoting poor nutritional messages to children. J Obes Weight Loss Ther 2013;4:212.

4. Calvert SL. Children as consumers: Advertising and marketing. Futur Child 2008;1:205-234.

5. Rideout V. Zero to eight: Children's media use in America 2013: a common sense media research study. Available at https://www.commonsensemedia.org/research/zero-to-eightchildrens-media-use-in-america-2013. Accessed December 14, 2017.

6. Lemish D. Children and Media: A Global Perspective. Maulden, MA: John Wiley \& Sons; 2015.

7. Katz E, Liebes T. Interacting with "Dallas" cross cultural readings of American TV. Can J Commun 1990;15:45-66.

8. Calvert SL, Kondla TA, Ertel KA, Meisel DS. Young adults' perceptions and memories of a televised woman hero. Sex Roles 2001;45:31-52.

9. Tian Q, Hoffner CA. Parasocial interaction with liked, neutral, and disliked characters on a popular TV series. Mass Commun Soc 2010;13:250-269. 
10. Chory RM. Differences in television viewers' involvement: Identification with and attraction to liked, disliked, and neutral characters. Commun Res Reports 2013;30:293-305.

11. Putnam MM, Cotto CE, Calvert SL. Character apps for children's snacks: effects of character awareness on snack selection and consumption patterns. Games Health J 2018; doi:10.1089/g4h.2017.0097.

12. Pempek T, Calvert SL. Tipping the balance: Use of advergames to promote consumption of nutritious foods and beverages by low-income African American children. Arch Pediatr Adolesc Med 2009;163:633-637.

13. Ogle AD, Graham DJ, Lucas-Thompson RG, Roberto CA. Influence of cartoon media characters on children's attention to and preference for food and beverage products. $\mathbf{J}$ Acad Nutr Diet 2017;117:265-270.

14. Kraak VI, Story M. Influence of food companies' brand mascots and entertainment companies' cartoon media characters on children's diet and health: A systematic review and research needs. Obes Rev 2015;16:107-126.

15. Baranowski T, Buday R, Thompson DI, Baranowski J. Playing for real: Video games and stories for health-related behavior change. Am J Prev Med 2008;34:74-82.

16. Roseberry S, Hirsh-Pasek K, Golinkoff RM. Skype me! Socially contingent interactions help toddlers learn language. Child Dev 2014;85:956-970.

17. Auty S, Lewis C. "The delicious paradox": Preconscious processing of product placements by children. In: The Psychology of Entertainment Media: Blurring the Lines between Entertainment and Persuasion, L.J. Strum, ed. Mawah, NJ: Lawrence Erlbaum; 2004:117-133.

18. Auty S, Lewis C. Exploring children's choice: The reminder effect of product placement. Psychol Mark 2004;21: 697-713.

19. Calvert SL, Tart M. Song versus verbal forms for very-longterm, long-term, and short-term verbatim recall. J Appl Dev Psychol 1993;14:245-260.
20. Waterman AH, Blades M, Spencer C, Waterman A. Do children try to answer nonsensical questions? Br Joumal Dev Psychol 2000;18:211-225.

21. Calvert SL. Developmental differences in children's production and recall of information as a function of computer presentational features. J Educ Comput Res 1994;10:139_ 151.

22. Calvert SL. Presentational features for young children's production and recall of information. J Appl Dev Psychol 1991;12:367-378.

23. Gujarati DJ, Porter DC. Qualitative response regression models. In: Basic Econometrics. 5th edit. New York: McGraw-Hill; 2009.

24. Watson WR, Mong CJ, Harris CA. A case study of the inclass use of a video game for teaching high school history. Comput Educ 2011;56:466-474.

25. Baranowski T, Blumberg F, Buday R, et al. Games for health for children-current status and needed research. Games Health J 2016;5:1-12.

26. Byrne S, Gay G, Pollack JP, et al. Caring for mobile phonebased virtual pets can influence youth eating behaviors. J Child Media 2012;6:83-99.

27. Hendrikse JJ, Cachia RL, Kothe EJ, et al. Attentional biases for food cues in overweight and individuals with obesity: A systematic review of the literature. Obes Rev 2015;16:424-432.

Address correspondence to: Marisa M. Putnam, MPP Children's Digital Media Center Georgetown University 313 White-Gravenor Hall 37 th and $O$ Streets, N.W. Washington, DC 20057

E-mail: mp1265@georgetown.edu 